\title{
Podocnemis expansa (Schweigger, 1812) (Reptilia, Testudines, Podocnemidae): espécie exótica no estado de Pernambuco, Nordeste, Brasil Podocnemis expansa (Schweigger, 1812) (Reptilia, Testudines, Podocnemidae): exotic species in the State of Pernambuco, Northeast Region of Brazil
}

\author{
Ederjow dos Santos', Luciana Carla Rameh-de-Albuquerque", \\ Alexandre Pinheiro Zanotti"l', Edivania do Nascimento Pereiral', Ednilza Maranhão dos Santos \\ Universidade Federal Rural de Pernambuco. Recife, Pernambuco, Brasil \\ "Ecoassociados. Ipojuca, Pernambuco, Brasil \\ "IICentro Operacional Brasileiro de Répteis e Aracnídeos. Rio Formoso, Pernambuco, Brasil
}

\begin{abstract}
Resumo: Em junho e dezembro de 2013, foram registrados, por meio de observação direta em uma Unidade de Conservação localizada no município de Recife, estado de Pernambuco, indivíduos adultos e desova de tartaruga-da-amazônia (Podocnemis expansa), espécie exótica para a região.
\end{abstract}

Palavras-chave: Testudines. Ocupação. Unidade de Conservação. Tartaruga-da-amazônia.
Abstract: In June and December 2013, the presence of adult individuals of the Amazon turtle (Podocnemis expansa) and their spawning were attested through direct observation, in a protected area located in the municipality of Recife, state of Pernambuco, Brazil, which is a region where the species is exotic.

Keywords: Testudines. Occupation. Conservation Unit. Amazon turtle.

SANTOS, E., L. C. RAMEH-DE-ALBUQUERQUE, A. P. ZANOTTI, E. N. PEREIRA \& E. M. SANTOS, 2016. Podocnemis expansa (Schweigger, 1812) (Reptilia, Testudines, Podocnemidae): espécie exótica no estado de Pernambuco, Nordeste, Brasil. Boletim do Museu Paraense Emílio Goeldi. Ciências Naturais 10(2): 261-265.

Autor para correspondência: Ednilza Maranhão dos Santos. Av. Dom Manoel de Medeiros, s/n - Dois Irmãos. Recife, PE, Brasil. CEP 52171900 (ednilzamaranhao@gmail.com).

Recebido em 10/03/2015

Aprovado em 10/02/2016

Responsabilidade editorial: Mário Augusto G. Jardim

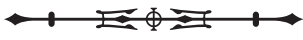




\section{INTRODUÇÃO}

A introdução acidental ou intencional de uma espécie fora de sua área de ocorrência natural caracteriza o organismo como espécie exótica. Esse organismo pode proporcionar um risco iminente às espécies nativas, como uma competidora desleal, não estando em seu habitat natural e sem seus predadores específicos, maximizando seu poder invasivo, podendo caracterizar-se também como uma espécie invasora (Sampaio \& Schmidt, 2013; Leão et al., 2011).

Para a fauna de tartaruga (Testudine) exótica em área natural no Brasil, há registro de duas espécies, que também são caracterizadas como invasoras, ambas pertencentes à Trachemys Agassiz, 1857, e com registro reprodutivo em Unidade de Conservação (Tortato et al., 2014), o que é proibido pela Lei Federal n 9.985/00 (Sampaio \& Schmidt, 2013). Na região Nordeste, existe registro de uma espécie de Testudine exótico e invasor ocupando ambiente natural, Trachemys scripta (Schoepff, 1792), conhecida como tartaruga-de-orelha-vermelha (Leão et al., 2011).

Podocnemis expansa (Schweigger, 1812) é considerado o maior quelônio de água doce da América do Sul, ocorrendo nas bacias dos rios Amazonas e Orinoco na Colômbia, na Venezuela, na Guiana, no leste do Equador, no nordeste do Peru, no norte da Bolívia e no norte e centro-oeste do Brasil. Habita grandes rios e seus tributários de águas claras ou escuras, além de lagoas e lagos adjacentes a estes rios (Ernst \& Barbour, 1989; Reva et al., 1989). Tais animais têm suas atividades de vida altamente relacionadas a fatores ambientais (temperatura, hidroperíodo, tipo de corpo d'água e trocas gasosas) que influenciam no seu desenvolvimento, evidenciando a determinação sexual (Malvasio, 2001). Na natureza, essa espécie é considerada herbívora, alimentando-se de cerca de 30 espécies de plantas pertencentes a 20 famílias (Vogt, 2008; Tratado de Cooperacion Amazonica, 1997). Em cativeiro é onívora, aceitando uma grande variedade de produtos vegetais, pescado e carne picada. Os filhotes mostram maior preferência por dieta carnívora quando comparados aos adultos (Alho et al., 1979; Tratado de Cooperacion Amazonica, 1997). São ovíparos, com um número médio de 80 a 100 ovos por ninho, apresentando período de incubação de cerca de dois meses em época seca (Vogt, 2008). Essa espécie encontra-se atualmente no Anexo II da Convenção da CITES (CITES, 2013), pelo seu comércio e seu uso, que obedecem as regras estabelecidas pela legislação brasileira.

$O$ presente trabalho vem registrar, pela primeira vez, a ocorrência de indivíduos adultos e desova de $P$. expansa fora da sua área de distribuição, em ambiente natural no Parque Estadual de Dois Irmãos, no município de Recife, estado de Pernambuco, Nordeste do Brasil, considerando mais uma espécie de Testudine exótica ao norte do rio São Francisco.

\section{MATERIAL E MÉTODOS}

Local de estudo: as observações ocorreram no Parque Estadual de Dois Irmãos (PEDI), uma Unidade de Conservação de Proteção Integral constituída de floresta atlântica urbana, localizada na região metropolitana, a noroeste da cidade do Recife, estado de Pernambuco, região Nordeste do Brasil, ao norte do rio São Francisco (Figura 1), em corpos d'água naturais. O PEDI possui uma área de aproximadamente 384,42 hectares, sendo 14 hectares destinados ao zoológico do Recife, com uma altitude aproximada de $19 \mathrm{~m}$, acima do nível do mar, estando entre as coordenadas geográficas $08^{\circ} 00^{\prime} 48.0^{\prime \prime}$ de latitude sul e 340 56' 42.9" de longitude oeste. Nesse remanescente de floresta úmida, encontram-se quatro principais recursos hídricos, que estão interligados entre si, os açudes do Prata, do Meio, de Dentro e Dois Irmãos, fazendo parte da bacia do Capibaribe (Machado et al., 1998).

Os registros ocorreram na primeira semana de junho, entre 13:00 e 17:00 horas, e no dia 29 de novembro 2013, por meio de observação direta, durante monitoramento de Testudines nos açudes de Dois Irmãos e de Dentro, ambos com conexão subterrânea, desaguando no rio Capibaribe. O açude Dois Irmãos (coordenadas -8.011048, -34.947212) 


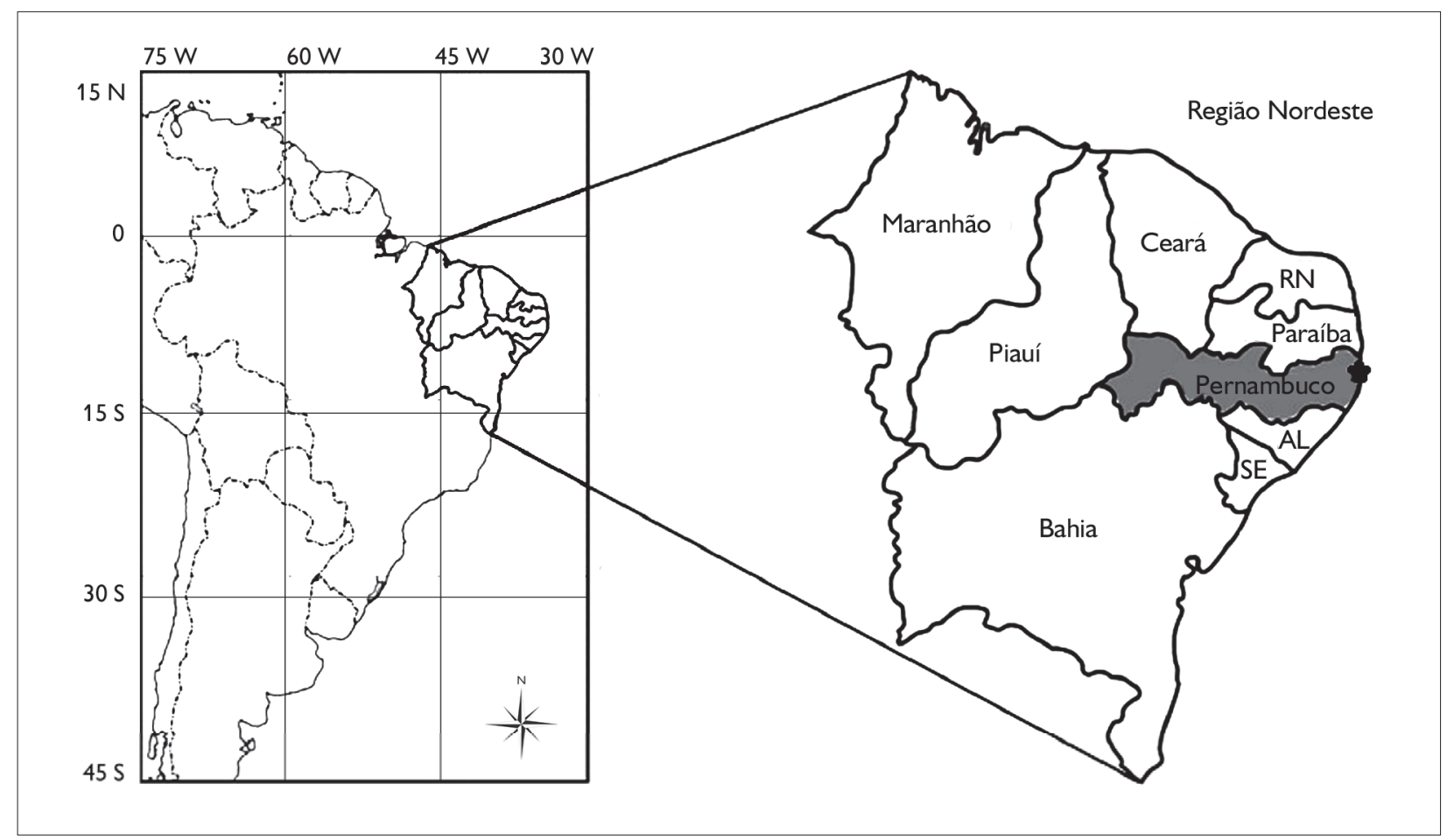

Figura 1. Localização do registro de Podocnemis expansa no Parque Estadual de Dois Irmãos, Recife, Pernambuco, Brasil. Fonte adaptada: Alves et al. (2008).

encontra-se bastante assoreado, com presença de pequenas ilhas flutuantes compostas principalmente por vegetação herbácea e pequenos arbustos. Limita-se com a Av. Dom Manoel de Medeiros e a Universidade Federal Rural de Pernambuco. $\bigcirc$ açude de Dentro (coordenadas -8.0105633, -34.9465978) tem conexão com o açude Dois Irmãos, sendo separados por uma estrada de acesso aos visitantes do zoológico e uma mureta de contenção; nele, há vegetação herbácea, com plantas flutuantes na lâmina d'água, além de vegetações emergentes, como algumas Poaceae; ambos representam 8,11\% da área do PEDI (Silvestre \& Carvalho, 1998; Machado et al., 1998). Utilizou-se, para registro dos animais, um binóculo e uma filmadora Nikon. As imagens estão depositadas no acervo da coleção Herpetológica da Universidade Federal Rural de Pernambuco. Utilizou-se, para identificação, as orientações de Ernst \& Barbour (1989) e Reva et al. (1989).

\section{RESULTADOS E DISCUSSÃO}

Adultos ( $n=3$; cerca de 80 a $90 \mathrm{~cm}$ de comprimento de carapaça) (Figura 2) foram observados em deslocamento e termorregulando entre a lâmina d'água dos açudes, geralmente entre os horários 13:00 e 17:00 horas, e em locais mais profundos (cerca de 5 a $7 \mathrm{~m}$ de profundidade), em temperatura variando de 28,5 a $29^{\circ} \mathrm{C}$. No dia 29/12/2013, uma desova com 47 ovos quebrados, possivelmente predados por lagartos pertencentes à família Teidae (Salvator merianae (Duméril \& Bibron, 1839) e/ou Ameiva ameiva Linnaeus, 1758), e oito ovos intactos foi registrada em solo argiloso/arenoso a 1,5 m da margem do açude de Dentro (Figuras 3 e 4). Os ovos que sobraram foram predados no dia seguinte.

Especula-se, com base em comunicação pessoal de técnicos do PEDI, que esse animal tenha sido introduzido no parque acidentalmente ou por meio de fuga de animais de cativeiro, como de recintos encontrados no Zoológico

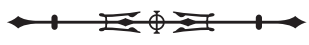


do próprio parque. A manutenção de animais em cativeiro no PEDI teve início em 1939, quando foi inaugurado o Horto Zoobotânico de Dois Irmãos, o qual só mudou de categoria para Parque Estadual de Dois Irmãos em 1998 (Machado et al., 1998). Não há documentação de quando os primeiros espécimes chegaram aos tanques do Zoológico, mas estima-se (de acordo com M. Falcão, comunicação pessoal) que essa espécie faz parte do plantel desde a formação do Horto Zoobotânico de Dois Irmãos.

No Nordeste do Brasil, especificamente nos estados da Paraíba e do Piauí, há registro de apenas um Testudine

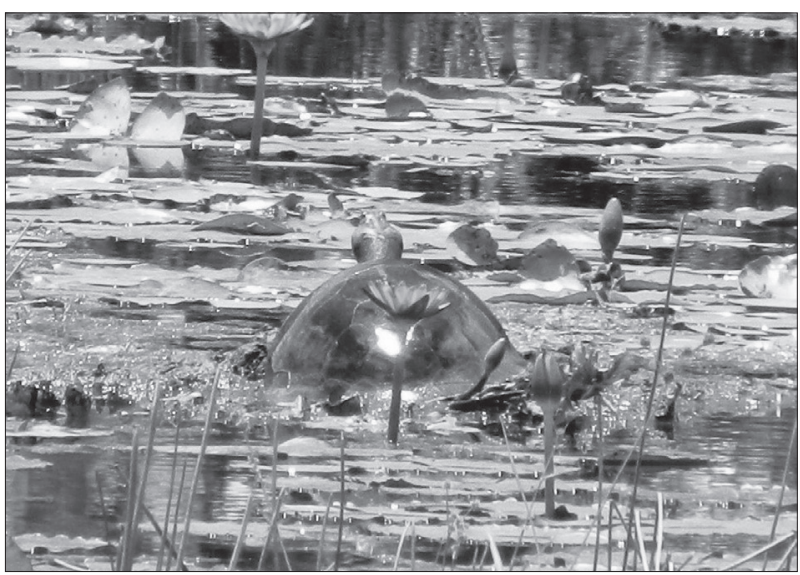

Figura 2. Podocnemis expansa, Parque Estadual de Dois Irmãos, Recife, Pernambuco, Brasil. Indivíduo adulto em comportamento de assoalhamento, registrado em 05/07/2013. Foto: Daliana Teles.

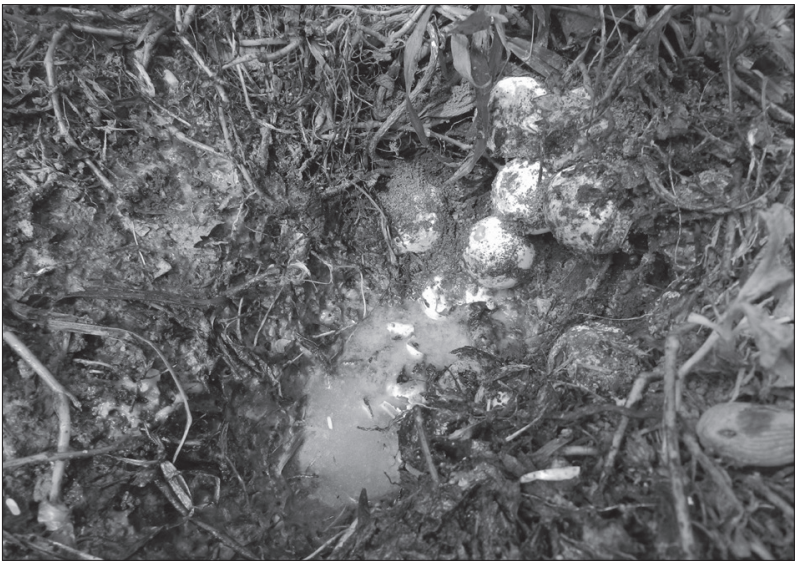

Figura 3. Desova de Podocnemis expansa, Parque Estadual de Dois Irmãos, Recife, Pernambuco, Brasil, em 29/12/2013. Foto: Ednilza M. Santos. exótico, considerado invasor e classificado como de risco médio, segundo Leão et al. (2011), os quais o definem como espécie exótica que ainda não foi reconhecida como invasora na região ou em outras regiões, ocorrendo em menos de três estados e possuindo menos de dez registros. Essa espécie, conhecida como tartaruga-de-orelhavermelha, Trachemys scripta, pertence à família Emydidae, tem sua origem no Vale do Mississipi, Estados Unidos, e foi introduzida em vários lugares do mundo através do comércio de animais de estimação (Bringsøe, 2006; Leão et al., 2011). Seus principais impactos são: ocupação de nichos de outras espécies de tartarugas, competição por alimento e espaço para assoalhamento e predação de outras espécies (Leão et al., 2011). É possível que impactos como competição por alimento e local de desova possam ocorrer também com a ocupação da $P$. expansa, aparentemente bem estabelecida no Parque Estadual de Dois Irmãos. Todavia, um programa de manejo, com monitoramento nas áreas de registro, deve fazer parte das ações dessa Unidade de Conservação.

Segundo o Sistema Nacional de Unidades de Conservação (Lei Federal nº 9.985/00; Brasil/Ministério do Meio Ambiente, 2000), é proibida a introdução de espécies exóticas em ambiente natural. As Unidades de Conservação, com base nos Sistemas Nacionais de

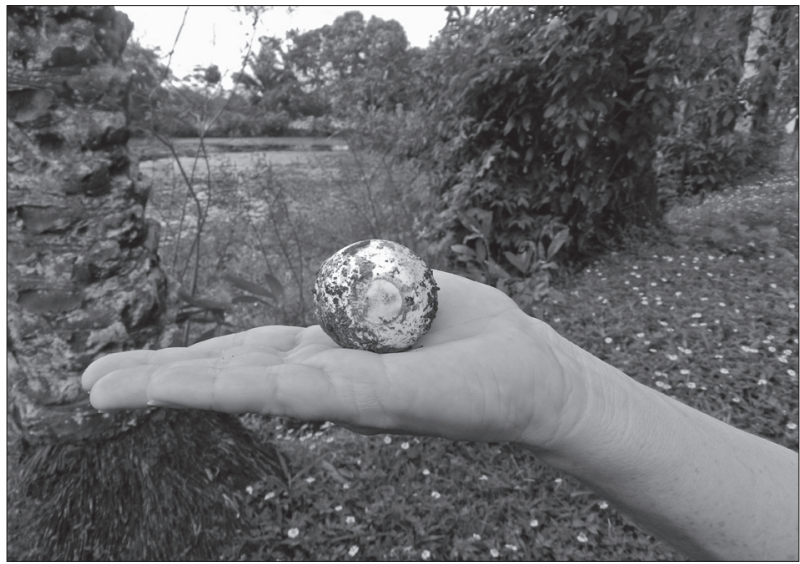

Figura 4. Ovo de Podocnemis expansa, Parque Estadual de Dois Irmãos, Recife, Pernambuco, Brasil, em 29/12/2013. Foto: Ednilza M. Santos

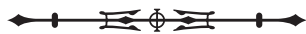


Unidades de Conservação da Natureza (art. 2 ${ }^{\circ}$ Brasil/ Ministério do Meio Ambiente, 2000), devem proteger a biodiversidade, prevenindo-a contra as ameaças, tomando medidas que desestimulem a introdução dessas espécies e/ou que retirem esses animais, principalmente de áreas de proteção integral. Espécies exóticas e invasoras causam prejuízos não só ao ambiente natural, mas também à economia e à saúde, podendo provocar impactos sociais e culturais (Leão et al., 2011). Medidas para erradicar espécies exóticas e invasoras em Unidades de Conservação devem ser urgentes.

\section{CONCLUSÃO}

Padocnemis expansa é um novo Testudine exótico em Unidade de Conservação na Mata Atlântica do nordeste do Brasil, ao norte do rio São Francisco.

\section{AGRADECIMENTOS}

À gestão do Parque Estadual de Dois Irmãos, pela autorização para realização desse estudo. Ao biólogo Igor Joventino Roberto, pela leitura do manuscrito e sugestões. À bióloga Marina Falcão e aos alunos do Laboratório de Herpetologia da Universidade Federal de Pernambuco.

\section{REFERÊNCIAS}

ALHO, C. J. R., A. G. CARVALHO \& L. F. M. PÁDUA, 1979. Ecologia da tartaruga da Amazônia e avaliação de seu manejo na reserva biológica do Trombetas. Brasil Florestal 38: 29-47.

ALVES, R. R. N., H. N. LIMA, M. C. TAVARES, W. M. S. SOUTO, R. R. D. BARBOZA \& A. VASCONCELLOS, 2008. Animal-based remedies as complementary medicines in Santa Cruz do Capibaribe, Brazil. BMC Complementary and Alternative Medicine 8(44): 1-9.

BRASIL/MINISTÉRIO DO MEIO AMBIENTE, 2000. Lei No 9985/2000 - "Regulamenta o art. 225, § 1o, incisos I, II, III e VII da Constituição Federal, institui o Sistema Nacional de Unidades de Conservação da Natureza e dá outras providências". Data da legislação: 18/07/2000. Diário Oficial da União, Brasília, 19 julho 2000. Disponível em: <http://www.mma.gov.br/port/conama/ legiabre.cfm?codlegi=322> . Acesso em: janeiro 2014.
BRINGSØE, H., 2006. Invasive alien species fact sheet - Trachemys scripta. NOBANIS. Disponível em: <https://www.nobanis.org/ fact-sheets/>. Accesso em: 13 novembro 2014.

CONVENTION ON INTERNATIONAL TRADE IN ENDANGERED SPECIES OF WILD FAUNAAND FLORA (CITES), 2013. Appendices I, II and III valid from 25 September 2012. Disponível em: <http:/hww.cites.org/eng/app/2012/E-2012-09-25. pdf $>$. Acesso em: 26 fevereiro 2013.

ERNST, C. H. \& R. W. BARBOUR, 1989. Turtles of the world: 1-313. Smithsonian Institution, Washington.

LEÃO, T. C. C., W. R. ALMEIDA, M. DECHOUM \& S. R. ZILLER, 2011. Espécies exóticas invasoras no Nordeste do Brasil: contextualização, manejo e políticas públicas: 1-99. CEPAN/Instituto Hórus, Recife.

MACHADO, I. C., A. V. LOPES \& K. C. PÔRTO, 1998. Reserva Ecológica de Dois Irmãos: estudos em um remanescente de Mata Atlântica em área urbana: 1-608. Editora Universitária UFPE, Recife.

MALVASIO, A., 2001. Aspectos do mecanismo alimentar e da biologia reprodutiva em Podocnemis expansa (Schweigger, 1812), Podocnemis unifilis (Troschel, 1848) e P. sextuberculata (Cornalia, 1849) (Testudines, Pelomedusidae): 1-199, Tese (Doutorado em Zoologia) - Universidade de São Paulo, São Paulo.

REVA, E., M. BALENSIEFER \& R. H. B. SILVA, 1989. Projeto Quelônios da Amazônia: 10 anos: 1-119. IBAMA, Brasília.

SAMPAIO, A. B. \& I. B. SCHMIDT, 2013. Espécies exóticas invasoras em Unidades de Conservação federais do Brasil. Biodiversidade Brasileira 3(2): 32-49.

SILVESTRE, C. W. \& P. V. CARVALHO, 1998. Bacia do Prata: aspectos qualitativos da água. In: I. C. MACHADO, A. V. LOPES \& K. C. PÔRTO (Eds.): Reserva ecológica de Dois Irmãos: estudos em um remanescente de Mata Atlântica em área urbana: 51-64. Editora Universitária UFPE, Recife.

TORTATO, M. A., R. F. BRESSAN \& T. S. KUNZ, 2014. Reproduction of two exotic species of Trachemys Agassiz, 1857 (Testudines, Emydidae) at Parque Estadual da Serra do Tabuleiro, state of Santa Catarina, southern Brazil. Herpetology Notes 7: 11-15.

TRATADO DE COOPERACION AMAZONICA, 1997. Biologia y manejo de la tortuga Podocnemis expansa (Testudines, Pelomedusidae): 1-48. Secretaria Pro Tempore, Caracas, Venezuela.

VOGT, R. C., 2008. Tartarugas da Amazônia: 1-104. Gráfica Biblos, Lima.

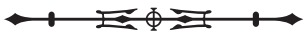


